\title{
Geographie im Dienste der Raumplanung
}

Vom 7. -13. Sept. 1975 führte die Kommission der angewandten Geographie der IGU (Internationale Geographische Union) in Liège (Belgien) ein einwöchiges Kolloquium mit dem Thema:

"Urbanisme commercial et renovation u rbaine" durch. Geographen aus verschiedenen Ländern stellten ihre Arbeiten zu diesem Themenkreis vor. Die nachfolgenden Aeusserungen beziehen sich nicht auf die einzelnen Referate, sonder'n skizzieren einige Ueberlegungen, die ich während des Kolloquiums und während der nachfolgenden Tage über die Arbeit der Geographen im Zusammenhang mit der Raumplanung anstellte.

Der grösste Teil der vorgelegten Arbeiten erschöpfte sich in Analysen von bestehenden $\mathrm{Zu}$ ständen und im Nachvollzug bisheriger räum licher Entwicklungen. Das Sammeln von Daten über die einzelnen Stadtelemente stand im Vordergrund. $\mathrm{Zu}$ einzelnen Gegenständen wie Verteilung der Bevölkerung nach Altersgruppen, Ausmass der Verkehrsströme usw. lagen graphisch saubere Karten vor. Die Referate fanden im allgemeinen ihren Abschluss mit dem Hinweis, dass diese Karten der Planung zur Verfügung gestellt wurden. Doch die Erstellung von schönen Karten allein, ist für mich noch nicht ausreichend, um stolz auf die Arbeit des Geographen zu sein. Vielmehr interessiert mich die Frage, was denn mit der vom Geographen aufgearbeitetenInformation geschieht, und wie die Daten tatsächlich eine Verwertung erfahren.

Die Untersuchung bestehender Zustände oder bisheriger Entwicklungen kann ausserordentlich wichtig sein, wenn es darum geht, herauszufinden, welche Beziehungen zwischen den einzelnen Elementen des räumlichen Gefüges bestehen oder bestanden haben. Erstaunlicherweise beschränkte sich aber ein Grossteil der vorgestellten geographischen Arbeiten auf die Aneinanderreihung von Einzelinformationen. Auf das Wirkungsgefüge wird jedoch nur selten eingegangen. Die Untersuchung von Wirkungsgefügen wäre aber gerade deshalb von grosser Bedeutung, weil im Rahmen der Raumplanung jeweils Massnahmen zur Erreichung gewisser Ziele bezüglich einer räumlichen Ordnung vor geschlagen werden. Doch Massnahmen vorzuschlagen, ohne genauere Kenntnisse über die möglichen Wirkungen zu haben, erscheint als nicht verantwortbar, ja geradezu als fahrlässig (und aus diesem Grunde ist es nicht erstaunlich, dass es ab und zu Fehlplanungen gibt! ).

Obwohl die Situation im Bereiche des menschlichen Handelns wesentlich komplizierter ist, sei ein Vergleich mit der Situation in der Medizin vorgenommen. Der menschliche Körper wurde im Verlaufe der Zeit dahin untersucht, welche Wirkungszusammenhänge z. B. zwischen den einzelnen Organen bestehen. Aus der Analyse kristallisierte sich ein Gefüge heraus, welches als Normalzustand zu bezeichnen ist. Abweichungen von diesem Normalzustand nennt man Krankheit. Als entsprechenden Ausdruck für die Raumplanung könnte "Problem" gesetzt werden. Die Erfolge der Medizin haben meiner Ansicht nach ihre Wurzeln in der soliden Kenntnis des Wirkungs gefüges des menschlichen Organismus.

Wie gesagt, die Situation im Bereiche des Handelns und Verhaltens des Menschen im Raum ist wesentlich komplizierter, weil die Vorgänge nicht determiniert sind. Ferner kommt hinzu, dass ein Normalzustand nicht vorgegeben ist, sondern durch einen Meinungsbildungsprozess jeweils erst umschrieben werden muss. Wir stellen fest, dass heute die Kenntnisse über räumliche Wirkungs gefüge noch weitgehend fehlen, und trotzdem werden täglich Massnahmen getroffen, welche die räumliche Ordnung verändern. Die Situation der Geographie und der Raumplanung scheint mir vergleichbar mit der Situation in den Anfängen der Heilkunst: Man verabreichte dem Patienten ein Heilmittel und hoffte dann vor allem, dass sich eine positive Wirkung einstellen werde. Es ist meiner Ansicht nach Aufgabe der Geographie zuhanden der Raumplanung Wirkungsgefüge in räumlichen Ordnungen aufzudecken. Die Tatsache, dass die Verhältnisse sehr kompliziert sind, sollte nicht als Entschuldi gung, sondern als Aufforderung zur Inangriffnahme der Arbeit verstanden werden.

Dr. Peter Gresch, Oberassistent am ORLInstitut der ETHZ, Gebäude Hil, Hönggerberg, 8093 Zürich. 
An und für sich ist es nicht erstaunlich - um zu unserem Vergleichsbild zurückzukehren dass die Medizin ihr Wissen auf diesem weit fortgeschrittenen Stand hat, arbeiteten doch schon Generationen von Medizinern daran, das Wirkungsgefüge des menschlichen Körpers zu erforschen. Zur Untersuchung des Wirkungsgefüges urbaner und ländlicher Räume steht indessen lediglich eine kleine Zahl von Geographen und Raumplaner im Einsatz.

Die heutige Situation in der Geographie kann durch folgendes Erlebnis illustriert werden: Im Rahmen des Kolloquiums in Liege machten wir einen Besuch beim Stadtplanungsamt in Brüssel. Im Ausstellungsraum des Amtes waren eine grosse Zahl von sogenannten Grundlagekarten aufgehängt: Entwicklung der Bodenpreise für das Bauen, Bodenbeschaffenheit, Lage und Grösse der Schulhäuser usw. Insgesamt waren es nahezu zwanzig Karten. Daneben war auch der Zonenplan (plan de secteur) aufgehängt. Wie man sich so die Raumplanung vorstellt, war ich der Auffassung, dass die Grundlagekarten effektiv die Grundlage für die Erstellung des Zonenplanes bilden würden. Es war jedoch nicht auf Anhieb ersichtlich, wie diese Grundlageinformationen zum resultierenden Zonenplan verarbeitet wurden. Ich erkundigte mich deshalb bei einem Vertreter des Stadtplanungsamtes nach der Methode, welche für den Zusammenbau gewählt wurde und erhielt folgende Auskunft: "Dies ist eine sehr interessante Frage (!); wir gehen eben jetzt daran zu untersuchen, wie diese Grundlage informationen zusammengebaut und zum Zonenplan übergeführt werden könnten"; - und zwei Meter neben dem Mann hing bereits der rechtsgültige Zonenplan! Mich packte ein Unbehagen, weil ich mir vorstellte, mit welcher Sorgfalt die Geographen die Grundlagekarten hergestellt hatten.

Ich dachte über diese Begebenheit nach und fragte mich, weshalb es zu einer derartigen Missachtung der geographischen Arbeit kommen konnte. Ich bin zur Auffassung gekommen, dass dieser Gang der Dinge gar nicht überraschend ist, denn die Geographen haben sich wahrscheinlich auch hier damit zufrieden gegeben, dass sie schöne Karten hergestellt hatten. Sie haben nicht gefragt, was mit diesen Informationen geschieht und deshalb auch keine Verfahren entwickelt, welche sie zur Synthese anbieten könnten.

In diesem Bereich haben wir Geographen noch ein grosses Stück Arbeit zu leisten. Wesentlich scheint mir, dass vorerst die Probleme und die daraus resultierenden Arbeiten überhaupt erst gesehen werden.

Am Kolloquium in Liège gewann ich den Eindruck, dass sich der Geograph zu gern auf das stürzt, was offensichtlich ist und sich leicht in eine Karte umsetzen lässt. Die aktuelle Situation verlangt aber von der Geographie mehr Tiefgang, denn die Raumplanung ist dringend auf Kenntnis räumlicher Wirkungsgefüge angewiesen. 